Bull. Austral. Math. Soc.

VOL. 58 (1998) [465-468]

\title{
PROOFS OF SOME HYPERELLIPTIC FUNCTION IDENTITIES
}

\section{Michael D. HiRsChHORN}

We give simple direct proofs of some hyperelliptic function identities conjectured by R.J. Baxter which arose in his study of the chiral Potts model.

\section{INTRODUCTION}

In a recent paper [1], Baxter defines

$$
\begin{aligned}
\Phi(\alpha, \beta) & =\sum_{m, n=-\infty}^{\infty} x^{m^{2}+m n+n^{2}} \alpha^{m} \beta^{n}, \\
\Phi_{j}(\alpha, \beta) & =\sum_{m-n \equiv j(\bmod 3)} x^{m^{2}+m n+n^{2}} \alpha^{m} \beta^{n}, j=0,1,2 .
\end{aligned}
$$

He then defines

$$
V(x)=\Phi(1,1), \quad V_{j}(x)=\Phi_{j}(1,1), \quad j=0,1,2 .
$$

He states various relations involving the $V$ 's, and shows that they follow from results in [2]. It seems desirable to present proofs of his relations, as direct and simple as possible, and that is what we shall attempt to do here.

The relations we shall prove are as follows. $Q(x)=\prod_{n \geqslant 1}\left(1-x^{n}\right)$ is Baxter's notation.

$$
\begin{gathered}
V_{2}(x)=V_{1}(x), \\
V_{0}(x)=V\left(x^{3}\right) \\
V_{0}(x)-V_{1}(x)=Q(x)^{3} / Q\left(x^{3}\right), \\
V_{0}(x)^{3}-V_{1}(x)^{3}=Q\left(x^{3}\right)^{9} / Q\left(x^{9}\right)^{3} \\
V(x)=1+6 \sum_{n \geqslant 1}\left(\frac{x^{3 n-2}}{1-x^{3 n-2}}-\frac{x^{3 n-1}}{1-x^{3 n-1}}\right), \\
V_{1}(x)=V_{2}(x)=3 x Q\left(x^{9}\right)^{3} / Q\left(x^{3}\right) .
\end{gathered}
$$

and

$$
\Phi(x, x)=3 Q\left(x^{3}\right)^{3} / Q(x) .
$$

We shall see that (1) is trivial, (2) follows easily from the definitions, (4),(5) and (6) follow easily from (3) and (7) follows easily from (6); (3) is a little harder.

Received 30th March, 1998

Copyright Clearance Centre, Inc. Serial-fee code: 0004-9729/98 \$A2.00+0.00. 


\section{PROOFS}

We have

$$
\begin{aligned}
V_{2}(x) & =\sum_{m-n \equiv 2(\bmod 3)} x^{m^{2}+m n+n^{2}}=\sum_{m-n \equiv-1(\bmod 3)} x^{m^{2}+m n+n^{2}} \\
& =\sum_{n-m \equiv 1(\bmod 3)} x^{m^{2}+m n+n^{2}}=\sum_{m-n \equiv 1(\bmod 3)} x^{n^{2}+n m+m^{2}} \\
& =\sum_{m-n \equiv 1(\bmod 3)} x^{m^{2}+m n+n^{2}} \\
& =V_{1}(x) .
\end{aligned}
$$

This establishes (1).

We have

$$
V_{0}(x)=\sum_{m \equiv n(\bmod 3)} x^{m^{2}+m n+n^{2}} .
$$

Put $k=(m-n) / 3, \quad l=(-2 m-n) / 3$. Then $m=k-l, \quad n=-2 k-l$, $m^{2}+m n+n^{2}=3 k^{2}+3 k l+3 l^{2}$ and

$$
V_{0}(x)=\sum\left(x^{3}\right)^{k^{2}+k l+l^{2}}=V\left(x^{3}\right) .
$$

This is (2).

We now prove (3). We have, with $\omega^{3}=1, \omega \neq 1$,

$$
\begin{aligned}
& V_{0}(x)-V_{1}(x)=V_{0}(x)+\left(\omega+\omega^{2}\right) V_{1}(x) \\
&=V_{0}(x)+\omega V_{1}(x)+\omega^{2} V_{2}(x) \\
&=\sum \omega^{m-n} x^{m^{2}+m n+n^{2}} \\
&=\sum_{m+n+p=0} \omega^{m-n} x^{\left(m^{2}+n^{2}+p^{2}\right) / 2} \\
&=C T_{a}\left\{\sum_{-\infty}^{\infty} a^{m} \omega^{m} x^{m^{2} / 2} \sum_{-\infty}^{\infty} a^{n} \omega^{-n} x^{n^{2} / 2} \sum_{-\infty}^{\infty} a^{p} x^{p^{2} / 2}\right\} \\
&=C T_{a}\left\{\prod_{n \geqslant 1}\left(1+a \omega x^{n-\frac{1}{2}}\right)\left(1+a^{-1} \omega^{-1} x^{n-\frac{1}{2}}\right)\left(1-x^{n}\right)\right. \\
& \cdot \prod_{n \geqslant 1}\left(1+a \omega^{-1} x^{n-\frac{1}{2}}\right)\left(1+a^{-1} \omega x^{n-\frac{1}{2}}\right)\left(1-x^{n}\right) \\
&\left.\cdot \prod_{n \geqslant 1}\left(1+a x^{n-\frac{1}{2}}\right)\left(1+a^{-1} x^{n-\frac{1}{2}}\right)\left(1-x^{n}\right)\right\}
\end{aligned}
$$


[3]

$$
\begin{aligned}
& =C T_{a}\left\{\prod_{n \geqslant 1}\left(1+a^{3} x^{3 n-\frac{3}{2}}\right)\left(1+a^{-3} x^{3 n-\frac{3}{2}}\right)\left(1-x^{n}\right)^{3}\right\} \\
& =Q(x)^{3} / Q\left(x^{3}\right) \cdot C T_{a}\left\{\prod_{n \geqslant 1}\left(1+a^{3} x^{3 n-\frac{3}{2}}\right)\left(1+a^{-3} x^{3 n-\frac{3}{2}}\right)\left(1-x^{3 n}\right)\right\} \\
& =Q(x)^{3} / Q\left(x^{3}\right) \cdot C T_{a}\left\{\sum_{-\infty}^{\infty} a^{3 n} x^{3 n^{2} / 2}\right\} \\
& =Q(x)^{3} / Q\left(x^{3}\right) .
\end{aligned}
$$

Next we have

$$
V_{0}(x)-V_{1}(x)=Q\left(x^{3}\right)^{2} \prod_{n \geqslant 1}\left(1-x^{3 n-2}\right)^{3}\left(1-x^{3 n-1}\right)^{3} .
$$

Putting $x, \omega x, \omega^{2} x$ for $x$ and multiplying the three results gives (noting that $V_{0}(x)$ contains only powers of $x$ congruent to 0 modulo 3 and $V_{1}(x)$ contains only powers of $x$ congruent to 1 modulo 3 )

$$
\begin{aligned}
V_{0}(x)^{3}-V_{1}(x)^{3} & =Q\left(x^{3}\right)^{6} \prod_{n \geqslant 1}\left(1-x^{9 n-6}\right)^{3}\left(1-x^{9 n-3}\right)^{3} \\
& =Q\left(x^{3}\right)^{9} / Q\left(x^{9}\right)^{3}
\end{aligned}
$$

which is (4).

Again from (3) we have

$$
\begin{aligned}
V_{0}(x)-V_{1}(x) & =\frac{1}{Q\left(x^{3}\right)} \sum_{n \geqslant 0}(-1)^{n}(2 n+1) x^{\left(n^{2}+n\right) / 2} \\
& =\frac{1}{Q\left(x^{3}\right)}\left\{\sum_{-\infty}^{\infty}(-1)^{n}(6 n+1) x^{3\left(3 n^{2}+n\right) / 2}\right. \\
& \left.\quad-3 x \sum_{n \geqslant 0}(-1)^{n}(2 n+1) x^{9\left(n^{2}+n\right) / 2}\right\} .
\end{aligned}
$$

It follows that (see note above)

$$
V_{0}(x)=\frac{1}{Q\left(x^{3}\right)} \sum_{-\infty}^{\infty}(-1)^{n}(6 n+1) x^{3\left(3 n^{2}+n\right) / 2}
$$

and

$$
V_{1}(x)=\frac{1}{Q\left(x^{3}\right)} \cdot 3 x \sum_{n \geqslant 0}(-1)^{n}(2 n+1) x^{9\left(n^{2}+n\right) / 2}=3 x Q\left(x^{9}\right)^{3} / Q\left(x^{3}\right)
$$


The second of these is (6). The first gives, by virtue of (2),

$$
\begin{aligned}
V(x) & =\frac{1}{Q(x)} \sum_{-\infty}^{\infty}(-1)^{n}(6 n+1) x^{\left(3 n^{2}+n\right) / 2} \\
& =\frac{1}{Q(x)}\left[\frac{d}{d a}\left\{\sum_{-\infty}^{\infty}(-1)^{n} a^{6 n+1} x^{\left(3 n^{2}+n\right) / 2}\right\}\right]_{a=1} \\
& =\frac{1}{Q(x)}\left[\frac{d}{d a}\left\{a \prod_{n \geqslant 1}\left(1-a^{6} x^{3 n-1}\right)\left(1-a^{-6} x^{3 n-2}\right)\left(1-x^{3 n}\right)\right\}\right]_{a=1} \\
& =1+6 \sum_{n \geqslant 1}\left(\frac{x^{3 n-2}}{1-x^{3 n-2}}-\frac{x^{3 n-1}}{1-x^{3 n-1}}\right)
\end{aligned}
$$

which is (5).

Finally,

$$
V_{1}(x)=\sum_{m-n \equiv 1(\bmod 3)} x^{m^{2}+m n+n^{2}}
$$

Put $k=(m-n-1) / 3, \quad l=(m+2 n-1) / 3$. Then $m=2 k+l+1, \quad n=-k+l$, $m^{2}+m n+n^{2}=3 k^{2}+3 k l+3 l^{2}+3 k+3 l+1$ and

$$
V_{1}(x)=x \sum\left(x^{3}\right)^{k^{2}+k l+l^{2}+k+l}
$$

(7) now follows from (6).

\section{REFERENCES}

[1] R.J. Baxter, 'Some hyperelliptic function identities that occur in the chiral Potts model', J. Phys. A (submitted).

[2] J.W. Borwein, P.B. Borwein and F.G. Garvan, 'Some cubic modular identities of Ramanujan', Trans. Amer. Math. Soc. 343 (1994), 35-39.

School of Mathematics

UNSW

Sydney 2052

Australia 